\title{
Strengthening primary care in Hong Kong: fostering continuity of care from a health system perspective
}

Margaret K Ho *, MSc Public Health

Department of Public Health and Policy, London School of Hygiene and Tropical Medicine, London, United Kingdom

*Corresponding author: margaretkayho@gmail.com

Hong Kong Med J 2020;26:543-5

https://doi.org/10.12809/hkmj198368

In 2018, Hong Kong was reported to have most efficient health system globally. ${ }^{1}$ However, there are large imbalances: while $90 \%$ of in-patient care is managed by the public sector, ${ }^{2} 70 \%$ of primary care consultations occur privately. ${ }^{3}$

The current primary care system in Hong Kong remains underdeveloped, fragmented, and inefficient: only $60 \%$ of the population have a regular care source. ${ }^{4}$ "Doctor-shopping" culture, and a disproportionate emphasis on acute episodic care, have limited the development of lasting patientprovider relationships. ${ }^{3}$ This is exacerbated by primary care providers' limited gatekeeping roles, leading to poor continuity of care in an overburdened public system. ${ }^{2}$ Furthermore, Hong Kong faces a rapidly ageing population with the proportion of population $\geq$ aged 65 expected to reach $30 \%$ by $2039 . .^{5}$

Health systems depending predominantly on primary care have better health outcomes, such as improved access to care and decreased avoidable mortality. ${ }^{6}$ Continuity of care is a crucial aspect of a good primary care system, assuring a patientprovider relationship that extends over time and beyond illness episodes. ${ }^{7}$

This commentary focuses on strategies to foster continuity, as part of strengthening Hong Kong's primary care system. Three relatively new interventions are examined to address the three types of continuity: relational, management, and informational. ${ }^{8}$

\section{Relational continuity: reviewing the Elderly Health Care Voucher Scheme}

Launched in 2009, the Elderly Health Care Voucher Scheme provides an annual allowance of HK\$2000, in the form of vouchers, for citizens aged $\geq 65$ years to use on private outpatient services. ${ }^{9}$ It aims to provide financial and social protection for elderly patients and to encourage them towards a regular source of care best suited to their needs. ${ }^{9}$

Although primary care is more frequently managed privately, most likely due to greater accessibility and shorter waiting times, it also incurs substantial out-of-pocket payments making it unaffordable for some. The Elderly Health Care Voucher Scheme, as a public-private partnership, restructures health financing equitably to provide monetary incentive for elderly patients to seek private providers. This reduces the burden on the public sector, not only at the primary level but also at higher levels of care owing to better gatekeeping. This also improves quality of service delivery. Private providers are vetted before being included into the scheme to ensure safety and quality. Currently, there are over 4000 providers covering all districts in the city, maximising service coverage and accessibility for elderly patients, who are often financially vulnerable. ${ }^{9}$

There has been some improvement in relational continuity, that is, sustaining lasting patient-provider relationships. Among users registered under the Electronic Health Records Sharing System (eHRSS) with multiple claims, $75 \%$ attended only one medical provider, accounting for approximately half of total eligible users registered on eHRSS. ${ }^{7}$ However, in a 2011 review, 69\% of users spent their vouchers on acute episodic curative services, and only $21 \%$ of total claims represented consultations for the monitoring of chronic conditions. ${ }^{10}$ In a separate study, $66 \%$ claimed that "the scheme did not change their health seeking behaviours on seeing public or private healthcare professionals".11

There needs to be more targeted incentives towards continuity of care. This could start straight from the enrolment process by encouraging patients to register with and maintain attendance at one trusted care provider. Since this health financing strategy was successful in attaining high utilisation, other possible modifications could be to devote a portion of the vouchers to be used on preventive care, as well as to have discounted consultation fees for patients if they continuously attend the same primary care provider. Although continuity may initially be based on monetary gain, it would hopefully foster a genuine, trusting patient-provider relationship in the long run, as providers would be more familiar with patients over time. This strategy would likely be helpful for patients with chronic illnesses who 
benefit greatly from a regular source of care. It would also be considerably advantageous for system load balance as care for chronic illnesses usually require more resources. To address budgetary concerns, the government recently expressed willingness to invest more funds, if needed, to improve the scheme. ${ }^{12}$ Regular monitoring would be needed to ensure the effectiveness of such a change.

\section{Management continuity: integrating the medical workforce in Community Health Centres}

Aside from improving private care, it is necessary to ensure that public care is well-suited to citizens' needs. In response to this, Hong Kong developed Community Health Centres (CHCs), public one-stop polyclinics. ${ }^{13}$ To date, three CHCs in populationdense districts are in operation, with plans for more. ${ }^{13}$ Management continuity is emphasised with the appropriate provision of complementary care types for consistency of care. ${ }^{7}$ This is particularly important for patients with chronic illnesses who may require a variety of services.

Members of the health workforce, including doctors, nurses, and allied health professionals, work together at $\mathrm{CHCs}$ to offer medical consultations, health risk assessments, and preventive care. ${ }^{13}$ Service delivery is tailored to community needs. For example, as diabetes is prevalent in Hong Kong, diabetic eye and foot checks are provided. ${ }^{14}$ Some $\mathrm{CHCs}$ have self-service stations for patients to take their own blood pressure, weight, and height, to increase patients' control over their own health. ${ }^{14}$

Having regular and convenient access to a $\mathrm{CHC}$ with a diverse scope of services will likely prompt patients to engage in primary care more frequently. Consistent monitoring of their health will also allow for any problems to be addressed immediately.

However, having different healthcare professionals working at a $\mathrm{CHC}$ does not necessarily mean that integration or interprofessional collaboration will automatically be achieved. One barrier is the lack of understanding within the workforce on the roles of each professional, especially of those in allied health fields. In fact, an interview with a local professional health organisation revealed that "...trainees don't understand the practice and role of other professionals (ie, allied health) ....' This may lead to fragmentation or duplication of services delivered.

To address this challenge, good governance is key. For smooth horizontal and vertical integration, there needs to be clear definition on the responsibilities of different actors, and how each functions as part of the health system. ${ }^{15}$ All actors should be held publicly accountable for their respective tasks to ensure a high standard of care. ${ }^{15}$ This guidance should be fostered during education and training to promote an appreciation for integration in the health workforce early on. Periodic reviews of $\mathrm{CHCs}$ would be necessary to ascertain that the appropriate care is being delivered efficiently. These reviews may be done by considering health outcome measures such as patient mortality and morbidity, admission and readmission rates, as well as patients' perspectives.

\section{Informational continuity: expanding the Electronic Health Records Sharing System}

Achieving relational and management continuity requires seamless information sharing which requires the availability and use of patient information, that is, information continuity. ${ }^{7}$ In 2016, Hong Kong launched the eHRSS, an electronic platform for public and private healthcare providers to access consenting patients' health records, including results of investigations as well as management plans. ${ }^{15}$ It aims to provide smooth information transfer so that practitioners can draw on patient records to coordinate care.

This technology has many advantages. Having a single reporting system with patient records readily available allows practitioners to respond to patients' needs immediately and decreases likelihood of repeated tests or treatments being done which can reduce efficiency. ${ }^{16} \mathrm{~A}$ smooth transition of care can be achieved if patients attend to multiple providers or change practitioners. By providing patients with access to their official health records, this allows for greater transparency and accountability in a system where information asymmetry is a challenge. On a community level, eHRSS supports disease surveillance for public health security. ${ }^{15}$

One of the biggest challenges for eHRSS is lack of uptake. Although it has been well-received and is relatively new, only $10 \%$ of the general population and $40 \%$ of medical professionals are registered (as of April 2018), with the latter citing high costs of implementation and training as reasons for nonparticipation. ${ }^{2}$ This lack of use greatly limits its effectiveness.

On lack of patient uptake, there is potential for eHRSS to provide a patient-centred platform. In addition to current access to their own records, this platform could include services for appointment booking and personalised information on disease management and prevention. Patients could also contribute to their own records by providing updates and questions before consultations. This could be preceded by appropriate training sessions for patients, especially elderly ones. In this way, providers would be able to review their input and provide more targeted and efficient care, which is 
crucial considering limited consultation times. By supporting patients to engage with practitioners and take an active role in their own health, this could encourage co-production of health using an innovative and patient-centred approach. This would hopefully attract more patients towards the eHRSS. For healthcare providers, the provision of free training could boost participation.

It is suggested that for every HK\$1.00 invested into primary care, $\mathrm{HK} \$ 8.40$ is saved in costs on acute care. ${ }^{2}$ Primary care reform, starting with improvements in continuity of care, is in the city's best interests. Such a reform would require strong stewardship that understands the complex needs of today's patients, promotes patient-provider coproduction of health in an integrated people-centred system, and acknowledges the interdependence of systemic elements in effective interventions. ${ }^{17}$ Ultimately, this will allow Hong Kong to further improve upon its current promising solutions, making it better equipped to address population health needs.

\section{Author contributions}

The author contributed to the concept or design of the study, acquisition of the data, analysis or interpretation of the data, drafting of the manuscript, and critical revision of the manuscript for important intellectual content. The author had full access to the data, contributed to the study, approved the final version for publication, and takes responsibility for its accuracy and integrity.

\section{Conflicts of interest}

The author has no conflicts of interest to disclose.

\section{Funding/support}

This commentary received no specific grant from any funding agency in the public, commercial, or not-for-profit sectors.

\section{References}

1. Miller LJ, Lu W. These are the economies with the most (and least) efficient health care. Bloomberg.com. 2019. Available from: https://www.bloomberg.com/news/ articles/2018-09-19/u-s-near-bottom-of-health-indexhong-kong-and-singapore-at-top. Accessed 15 Feb 2019.

2. Our Hong Kong Foundation. Fit for Purpose: a health system for the 21st century. 2018. Available from: https:// www.ourhkfoundation.org.hk/sites/default/files/media/ pdf/ohkf_research_report_digital_1201.pdf. Accessed 15 Feb 2019.

3. Food and Health Bureau, Hong Kong SAR Government. Our partner for better health-primary care development in Hong Kong: strategy document. December 2010. Available from: https://www.fhb.gov.hk/download/press_ and_publications/otherinfo/101231_primary_care/e_ strategy_doc.pdf. Accessed 15 Feb 2019.
4. Wong SY, Zou D, Chung RY, et al. Regular source of care for the elderly: a cross-national comparative study of Hong Kong with 11 developed countries. J Am Med Dir Assoc 2017;18:807.e1-8.

5. Census and Statistics Department, Hong Kong SAR Government. Hong Kong Population Projections 20172066. 2019. Available from: https://www.statistics.gov.hk/ pub/B1120015072017XXXXB0100.pdf. Accessed 15 Feb 2019.

6. Starfield B, Shi L, Macinko J. Contribution of primary care to health systems and health. Milbank Q 2005;83:457-502.

7. Gulliford M, Naithani S, Morgan M. What is 'continuity of care'? J Health Serv Res Policy 2006;11:248-50.

8. Reid R, Haggerty J, McKendry R. Defusing the confusion: concepts and measures of continuity of health care. Ottawa: Canadian Health Services Research Foundation; 2002.

9. Health Care Voucher. Hong Kong SAR Government. Background of Elderly Health Care Voucher Scheme. 2019. Available from: https://www.hcv.gov.hk/eng/pub_ background.htm. Accessed 15 Feb 2019.

10. Food and Health Bureau, Department of Health, Hong Kong SAR Government. Interim review of Elderly Health Care Voucher Pilot Scheme. 2011. Available from: https:// www.hcv.gov.hk/files/pdf/ehcv_interim_review_report_ en.pdf. Accessed 15 Feb 2019.

11. Yam CH, Liu S, Huang OH, Yeoh EK, Griffiths SM. Can vouchers make a difference to the use of private primary care services by older people? Experience from the healthcare reform programme in Hong Kong. BMC Health Serv Res 2011;11:255.

12. News.gov.hk, Hong Kong SAR Government. Health voucher up for review. 2019. Available from: https://www. news.gov.hk/eng/2019/01/20190110/20190110_123219_0 08.html. Accessed 15 Feb 2019.

13. Primary Healthcare Office, Food and Health Bureau, Hong Kong SAR Government. Primary Healthcare Office. Available from: https://www.pco.gov.hk/english/ initiatives/centre.html. Accessed 15 Feb 2019.

14. South China Morning Post. Prevention better than cure: Kwun Tong Community Health Centre helping Hong Kong's growing elderly population stay one step ahead of chronic illness. Available from: https://www.scmp.com/ news/hong-kong/health-environment/article/1862165/ prevention-better-cure-kwun-tong-community-health. Accessed 15 Feb 2019.

15. World Health Organization. Everybody's businessstrengthening health systems to improve health outcomes: WHO's framework for action. Available from: https://apps. who.int/iris/handle/10665/43918. Accessed 15 Feb 2019.

16. Electronic Health Record Sharing System, Hong Kong SAR Government. What is eHR Sharing System? Available from: https://www.ehealth.gov.hk/en/about_ehrss/electronic_ health_record/what_is_ehrss.html. Accessed 15 Feb 2019.

17. World Health Organization Regional Office for Europe. Smart governance for health and well-being: the evidence. Available from: http://www.euro.who.int/_data/assets/ pdf_file/0005/257513/Smart-governance-for-health-andwell-being-the-evidence.pdf?ua=1. Accessed 15 Feb 2019. 